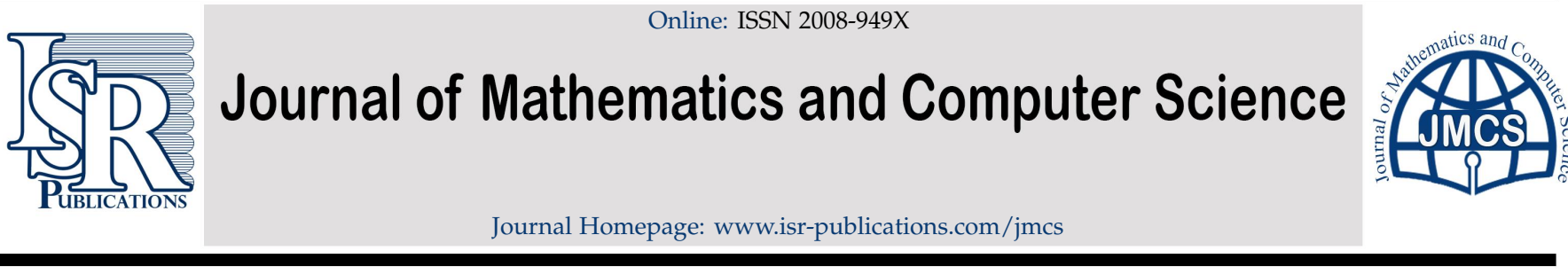

\title{
Estimation of f-divergence and Shannon entropy by Levin- son type inequalities for higher order convex functions via Taylor polynomial
}

\author{
Muhammad Adeel $^{a, b, *}$, Khuram Ali Khan ${ }^{a}$, Đilda Pečarićc ${ }^{\text {, Josip Pečarić }}{ }^{d}$ \\ ${ }^{a}$ Department of Mathematics, University of Sargodha, Sargodha-40100, Pakistan. \\ ${ }^{b}$ Department of Mathematics, University of Central Punjab, Lahore, Pakistan. \\ c University of Croatia, llica 242, Zagreb, Croatia. \\ ${ }^{d}$ RUDN University, Moscow, Russia.
}

\begin{abstract}
In this paper, Levinson-type inequalities are generalized by using Taylor polynomial for the class of $k$-convex ( $k \geqslant 3$ ) functions. Bounds for the remainders in new generalized identities involving data points of two types are given by using Čebyšev, Grüss and Ostrowski-type inequalities. In seek of applications of our results to information theory, new generalizations based on f-divergence estimates are also proven. Moreover, some inequalities for Shannon entropies are deduced as well.
\end{abstract}

Keywords: Information theory, convex functions, Levinson's inequality.

2020 MSC: 94A15, 94A17, 26D15.

(C)2020 All rights reserved.

\section{Introduction and Preliminaries}

The study of convex functions are used as a major tool to solve optimization problems in analysis. However the soil of inequalities involving convex functions is quite fertile as it increases the growth of many branches of mathematics with considerable high rate. That is why the study of such inequalities have been given great importance in literature (see [22]).

Divided differences are observed to be exceptionally useful when we are dealing with capacities having diverse degrees of smoothness. Definition of divided difference is given in [22, p. 14] as follows. The $\mathrm{k}^{\mathrm{th}}$-order divided difference of a function $\mathbf{f}:\left[\zeta_{1}, \zeta_{2}\right] \rightarrow \mathbb{R}$ at mutually distinct points $x_{0}, \ldots, x_{\mathrm{k}} \in\left[\zeta_{1}, \zeta_{2}\right]$ is defined recursively by

$$
\left[x_{\rho} ; \mathbf{f}\right]=\mathbf{f}\left(x_{\rho}\right), \quad \rho=0, \ldots, k, \quad\left[x_{0}, \ldots, x_{k} ; \mathbf{f}\right]=\frac{\left[x_{1}, \ldots, x_{k} ; \mathbf{f}\right]-\left[x_{0}, \ldots, x_{k-1} ; \mathbf{f}\right]}{x_{k}-x_{0}} .
$$

\footnotetext{
${ }^{*}$ Corresponding author

Email addresses: adeel.uosmaths@gmail.com (Muhammad Adeel), khuramsms@gmail.com (Khuram Ali Khan), gildapeca@gmail.com (Đilda Pečarić), pecaric@element.hr (Josip Pečarić)
}

doi: $10.22436 /$ jmcs.021.04.05

Received: 2019-10-12 Revised: 2020-02-07 Accepted: 2020-03-13 
It is easy to see that (1.1) is equivalent to

$$
\left[x_{0}, \ldots, x_{k} ; f\right]=\sum_{\rho=0}^{k} \frac{\mathbf{f}\left(x_{\rho}\right)}{q^{\prime}\left(x_{\rho}\right)}, \quad \text { where } q(x)=\prod_{j=0}^{k}\left(x-x_{j}\right) .
$$

The following definition of a real valued convex function is characterized by $k^{\text {th }}$-order divided difference (see [22, p. 15]).

Definition 1.1. A function $\mathbf{f}:\left[\zeta_{1}, \zeta_{2}\right] \rightarrow \mathbb{R}$ is said to be $k$-convex $(k \geqslant 0)$ if and only if for all choices of $(k+1)$ distinct points $x_{0}, \ldots, x_{k} \in\left[\zeta_{1}, \zeta_{2}\right],\left[x_{0}, \ldots, x_{k} ; f\right] \geqslant 0$ holds.

If this inequality is reversed, then $\mathbf{f}$ is said to be k-concave. If the inequality is strict, then $\mathbf{f}$ is said to be a strictly $k$-convex ( $k$-concave) function. Note that 0 -convex functions, 1 -convex functions, and 2convex functions are non-negative functions, increasing functions and are simply the convex functions, respectively. In [22, p. 16], criteria to examine the k-convexity of a function $\mathbf{f}$ is given as follows.

Theorem 1.2. If $\mathbf{f}^{(\mathrm{k})}$ exists, then $\mathbf{f}$ is $\mathrm{k}$-convex if and only if $\mathbf{f}^{(\mathrm{k})} \geqslant 0$.

In [15], (see also [18, p. 32, Theorem 1]) Ky Fan's inequality is generalized by Levinson for 3-convex functions as follows.

Theorem 1.3. Let $\mathbf{f}: I=(0,2 \alpha) \rightarrow \mathbb{R}$ with $\mathbf{f}^{(3)}(t) \geqslant 0, x_{k} \in(0, \alpha)$ and $p_{\rho}>0$. Then

$$
\frac{1}{P_{n}} \sum_{\rho=1}^{n} p_{\rho} f\left(x_{\rho}\right)-\mathbf{f}\left(\frac{1}{P_{n}} \sum_{\rho=1}^{n} p_{\rho} x_{\rho}\right) \leqslant \frac{1}{P_{n}} \sum_{\rho=1}^{n} p_{\rho} f\left(2 \alpha-x_{\rho}\right)-\mathbf{f}\left(\frac{1}{P_{n}} \sum_{\rho=1}^{n} p_{\rho}\left(2 \alpha-x_{\rho}\right)\right) .
$$

Working with the divided differences, assumptions of differentiability on $\mathbf{f}$ can be weakened.

In [23], Popoviciu noted that $(1.2)$ is valid on $(0,2 a)$ for 3-convex functions, while in [7], (see also [18, $\mathrm{p}$. 32, Theorem 2]) Bullen gave different proof of Popoviciu's result and also the converse of (1.2).

Theorem 1.4. Let $\mathbf{f}: \mathrm{I}=\left[\zeta_{1}, \zeta_{2}\right] \rightarrow \mathbb{R}$ be 3-convex function and $x_{\rho}, y_{\rho} \in\left[\zeta_{1}, \zeta_{2}\right]$ for $\rho=1,2, \ldots, n$ such that

$$
\max \left\{x_{1}, \ldots, x_{n}\right\} \leqslant \min \left\{y_{1}, \ldots, y_{n}\right\}, \quad x_{1}+y_{1}=\cdots=x_{n}+y_{n}
$$

and $\mathrm{p}_{\rho}>0$, then

$$
\frac{1}{P_{n}} \sum_{\rho=1}^{n} p_{\rho} f\left(x_{\rho}\right)-\mathbf{f}\left(\frac{1}{P_{n}} \sum_{\rho=1}^{n} p_{\rho} x_{\rho}\right) \leqslant \frac{1}{P_{n}} \sum_{\rho=1}^{n} p_{\rho} f\left(y_{\rho}\right)-\mathbf{f}\left(\frac{1}{P_{n}} \sum_{\rho=1}^{n} p_{\rho} y_{\rho}\right) .
$$

If $\mathbf{f}$ is continuous and $\mathrm{p}_{\rho}>0$, (1.4) holds for all $\mathrm{x}_{\rho}, \mathrm{y}_{\rho}$ satisfying (1.3), then $\mathrm{f}$ is 3 -convex.

Functional form of (1.4) is defined as follows:

$$
J(f(\cdot))=\frac{1}{P_{n}} \sum_{\rho=1}^{n} p_{\rho} f\left(y_{\rho}\right)-\mathbf{f}\left(\frac{1}{P_{n}} \sum_{\rho=1}^{n} q_{\rho} y_{\rho}\right)-\frac{1}{P_{n}} \sum_{\rho=1}^{n} p_{\rho} \mathbf{f}\left(x_{\rho}\right)+\mathbf{f}\left(\frac{1}{P_{n}} \sum_{\rho=1}^{n} p_{\rho} x_{\rho}\right) .
$$

Remark 1.5. It is important to note that under the assumptions of Theorem 1.4, if the function $\mathrm{f}$ is 3-convex then $J(f(\cdot)) \geqslant 0$ and $J(f(\cdot))=0$ for $\mathbf{f}(x)=x$ or $\mathbf{f}(x)=x^{2}$ or $\mathbf{f}$ is a constant function.

In [19], (see also [18, p. 32, Theorem 4]) Pečarić weakened the assumption (1.3) and proved that inequality (1.4) still holds, i.e., the following result holds.

Theorem 1.6. Let $\mathbf{f}: \mathrm{I}=\left[\zeta_{1}, \zeta_{2}\right] \rightarrow \mathbb{R}$ be a 3-convex function. Also, let $\mathrm{p}_{\rho}>0$ and $x_{\rho}, \mathrm{y}_{\rho} \in\left[\zeta_{1}, \zeta_{2}\right]$ be such that $x_{\rho}+y_{\rho}=2 \breve{c}$, for $\rho=1, \ldots, n, x_{\rho}+x_{n-\rho+1} \leqslant 2 \breve{c}$ and $\frac{p_{\rho} x_{\rho}+p_{n-\rho+1} x_{n-\rho+1}}{p_{\rho}+p_{n-\rho+1}} \leqslant \breve{c}$. The (1.4) holds. 
In [17], Mercer made a notable work by replacing the condition of symmetric distribution of points $x_{\rho}$ and $y_{\rho}$ with symmetric variances of points $x_{\rho}$ and $y_{\rho}$, second condition is weaker condition.

Theorem 1.7. Let $\mathbf{f}$ be a 3-convex function on $\left[\zeta_{1}, \zeta_{2}\right], p_{\rho}$ are positive such that $\sum_{\rho=1}^{n} p_{\rho}=1$. Also, let $x_{\rho}, y_{\rho}$ satisfy $\max \left\{x_{1} \ldots x_{n}\right\} \leqslant \min \left\{y_{1} \ldots y_{n}\right\}$ and

$$
\sum_{\rho=1}^{n} p_{\rho}\left(x_{\rho}-\sum_{\rho=1}^{n} p_{\rho} x_{\rho}\right)^{2}=\sum_{\rho=1}^{n} p_{\rho}\left(y_{\rho}-\sum_{\rho=1}^{n} p_{\rho} y_{\rho}\right)^{2}
$$

then (1.4) holds.

In [20], Pečarić et al. gave probabilistic version of Levinson's inequality (1.2) under Mercer's assumption of equal variances for the family of 3-convex functions at a point. They showed that this is the largest family of continuous functions for which the inequality (1.2) holds. Operator version of probabilistic Levinson's inequality discussed in [21].

Let us define the real valued function

$$
(x-t)_{+}= \begin{cases}(x-t), & t \leqslant x \\ 0, & t>x\end{cases}
$$

The Taylor's formula given in [6], as follows. Let $k$ be a positive integer and $\mathbf{f}:\left[\zeta_{1}, \zeta_{2}\right] \rightarrow R$ be such that $\mathbf{f}^{(k-1)}$ is absolutely continuous, then for all $x \in\left[\zeta_{1}, \zeta_{2}\right]$ the Taylor's formula at the point $c \in\left[\zeta_{1}, \zeta_{2}\right]$ is

$$
\mathbf{f}(x)=\mathrm{T}_{\mathrm{k}-1}(\mathbf{f} ; \mathrm{c}, \mathrm{x})+\mathrm{R}_{\mathrm{k}-1}(\mathbf{f} ; \mathrm{c}, \mathrm{x}),
$$

where $T_{k-1}(f ; c, x)$ is a Taylor's polynomial of degree $(k-1)$, i.e.,

$$
\mathrm{T}_{k-1}(\mathbf{f} ; \mathrm{c}, \mathrm{x})=\sum_{j=0}^{\mathrm{k}-1} \frac{\mathbf{f}^{(\mathbf{j})}(\mathrm{c})}{\mathrm{j} !}(x-\mathrm{c})^{j}
$$

and the remainder is given by

$$
R_{k-1}(f ; c, x)=\frac{1}{(k-1) !} \int_{c}^{x} \mathbf{f}^{(k)}(t)(x-t)^{k-1} d t .
$$

Applying Taylor's formula at the points $\zeta_{1}$ and $\zeta_{2}$, respectively, we get

$$
\begin{aligned}
\mathbf{f}(x) & =\sum_{j=0}^{k-1} \frac{\mathbf{f}^{(j)}\left(\zeta_{1}\right)}{j !}\left(x-\zeta_{1}\right)^{j}+\frac{1}{(k-1) !} \int_{\zeta_{1}}^{\zeta_{2}} \mathbf{f}^{(k)}(t)\left((x-t)_{+}^{k-1}\right) d t \\
\mathbf{f}(x) & =\sum_{j=0}^{k-1} \frac{\mathbf{f}^{(j)}\left(\zeta_{2}\right)}{j !}\left(\zeta_{2}-x\right)^{j}(-1)^{j}-\frac{1}{(k-1) !} \int_{\zeta_{1}}^{\zeta_{2}}(-1)^{k-1} \mathbf{f}^{(k)}(t)\left((t-x)_{+}^{k-1}\right) d t .
\end{aligned}
$$

In [8], Butt et al. generalized Popoviciu inequality via Taylor polynomial for higher order convex functions. Bounds, exponential convexity and Cauchy means were presented for linear functionals coming from the general inequality. All generalizations exist in litrature are only for one type of data points. But in this pattern and motivated by above discussion Levinson type inequalities are generalized via Taylor polynomial involving two type of data points for higher order convex functions. Čebyšev, Grüss and Ostrowski-type new bounds are also found for the functionals involving data points of two types.

\section{Main results}

Motivated by identity (1.5), we construct the following identities with help of (1.7) and (1.8), coming from Taylor polynomial. 
2.1. Bullen type inequalities for higher order convex functions

First we define the following functional $\mathcal{F}$ :

Let $\mathbf{f}: I=\left[\zeta_{1}, \zeta_{2}\right] \rightarrow \mathbb{R}$ be a function. Also, let $\left(\mathrm{p}_{1}, \ldots, \mathrm{p}_{\mathrm{n}}\right) \in \mathbb{R}^{\mathrm{n}}$ and $\left(\mathrm{q}_{1}, \ldots, \mathrm{q}_{\mathrm{m}}\right) \in \mathbb{R}^{\mathrm{m}}$ be such that $\sum_{\rho=1}^{n} p_{\rho}=1, \sum_{\rho=1}^{m} q_{\rho}=1$ and $x_{\rho}, y_{\rho}, \sum_{\rho=1}^{n} p_{\rho} x_{\rho}, \sum_{\rho=1}^{m} q_{\rho} y_{\rho} \in I$. Then

$$
\breve{J}(\mathbf{f}(\cdot))=\sum_{\rho=1}^{m} q_{\rho} \mathbf{f}\left(y_{\rho}\right)-\mathbf{f}\left(\sum_{\rho=1}^{m} q_{\rho} y_{\rho}\right)-\sum_{\rho=1}^{n} p_{\rho} \mathbf{f}\left(x_{\rho}\right)+\mathbf{f}\left(\sum_{\rho=1}^{n} p_{\rho} x_{\rho}\right) .
$$

Theorem 2.1. Assume $\mathcal{F}$ and $\mathbf{f}: \mathrm{I}=\left[\zeta_{1}, \zeta_{2}\right] \rightarrow \mathbb{R}$ be such that $\mathbf{f}^{(\mathrm{k}-1)}$ is absolutely continuous. Then (i)

$$
\breve{J}(\mathbf{f}(\cdot))=\breve{J}\left(T_{k-1}\left(\mathbf{f} ; \zeta_{1}, \cdot\right)\right)+\breve{J}\left(R_{k-1}\left(\mathbf{f} ; \zeta_{1}, \cdot\right)\right)
$$

where $\breve{\mathrm{J}}(\mathbf{f}(\cdot))$ is given by $(2.1)$,

$$
\begin{aligned}
\breve{\mathrm{J}}\left(\mathrm{T}_{k-1}\left(\mathbf{f} ; \zeta_{1}, \cdot\right)\right)= & \sum_{\rho=1}^{\mathrm{m}} \mathrm{q}_{\rho} \mathrm{T}_{k-1}\left(\mathbf{f} ; \zeta_{1}, \mathrm{y}_{\rho}\right)-\mathrm{T}_{k-1}\left(\mathbf{f} ; \zeta_{1}, \sum_{\rho=1}^{\mathrm{m}} \mathrm{q}_{\rho} \mathrm{y}_{\rho}\right) \\
& -\sum_{\rho=1}^{n} \mathrm{p}_{\rho} \mathrm{T}_{\mathrm{k}-1}\left(\mathbf{f} ; \zeta_{1}, x_{\rho}\right)+\mathrm{T}_{k-1}\left(\mathbf{f} ; \zeta_{1}, \sum_{\rho=1}^{n} \mathrm{p}_{\rho} \mathrm{x}_{\rho}\right)
\end{aligned}
$$

and

$$
\begin{aligned}
\breve{J}\left(R_{k-1}\left(\mathbf{f} ; \zeta_{1}, \cdot\right)\right)= & \sum_{\rho=1}^{m} q_{\rho} R_{k-1}\left(\mathbf{f} ; \zeta_{1}, y_{\rho}\right)-R_{k-1}\left(\mathbf{f} ; \zeta_{1}, \sum_{\rho=1}^{m} q_{\rho} y_{\rho}\right) \\
& -\sum_{\rho=1}^{n} p_{\rho} R_{k-1}\left(\mathbf{f} ; \zeta_{1}, x_{\rho}\right)+R_{k-1}\left(\mathbf{f} ; \zeta_{1}, \sum_{\rho=1}^{n} p_{\rho} x_{\rho}\right) .
\end{aligned}
$$

(ii)

$$
\breve{J}(\mathbf{f}(\cdot))=\breve{J}\left(T_{k-1}\left(\mathbf{f} ; \zeta_{2}, \cdot\right)\right)+\breve{J}\left(R_{k-1}\left(\mathbf{f} ; \zeta_{2}, \cdot\right)\right),
$$

where

$$
\begin{aligned}
\breve{\mathrm{J}}\left(\mathrm{T}_{k-1}\left(\mathbf{f} ; \zeta_{2}, \cdot\right)\right)= & \sum_{j=0}^{k-1} \frac{\mathbf{f}^{j}\left(\zeta_{2}\right)}{j !}(-1)^{j}\left(\sum_{\rho=1}^{\mathrm{m}} \mathrm{q}_{\rho}\left(\zeta_{2}-\mathrm{y}_{\rho}\right)^{j}-\left(\zeta_{2}-\sum_{\rho=1}^{\mathrm{m}} \mathrm{q}_{\rho} \mathrm{y}_{\rho}\right)^{j}\right. \\
& \left.-\left(\sum_{\rho=1}^{\mathrm{n}} \mathrm{p}_{\rho}\left(\zeta_{2}-x_{\rho}\right)^{j}\right)+\left(\zeta_{2}-\sum_{\rho=1}^{n} \mathrm{p}_{\rho} x_{\rho}\right)^{j}\right)
\end{aligned}
$$

and

$$
\begin{aligned}
\breve{J}\left(R_{k-1}\left(f ; \zeta_{2}, \cdot\right)\right)= & \frac{(-1)^{k-1}}{(k-1) !} \int_{\zeta_{1}}^{\zeta_{2}}\left(\sum_{\rho=1}^{m} q_{\rho}\left(t-y_{\rho}\right)_{+}^{k-1}-\left(t-\sum_{\rho=1}^{m} q_{\rho} y_{\rho}\right)_{+}^{k-1}\right. \\
& \left.-\sum_{\rho=1}^{n} p_{\rho}\left(t-x_{\rho}\right)_{+}^{k-1}+\left(t-\sum_{\rho=1}^{n} p_{\rho} x_{\rho}\right)_{+}^{k-1}\right) f^{(k)}(t) d t .
\end{aligned}
$$

Proof.

(i) Using (1.7) in (2.1) and by linearity of $\breve{J}(\cdot)$, we get (2.3). 
(ii) Same as proof of (i).

In the following theorem we obtain generalizations of Bullen type inequality for k-convex functions.

Theorem 2.2. Assume $\mathcal{F}$ and let $\mathbf{f}: \mathrm{I}=\left[\zeta_{1}, \zeta_{2}\right] \rightarrow \mathbb{R}$ be such that $\mathbf{f}^{(\mathrm{k}-1)}$ is absolutely continuous. Then

(i) if $\mathbf{f}$ is $\mathrm{k}$-convex function and

$$
\breve{J}\left(R_{k-1}\left(\mathbf{f} ; \zeta_{1}, \cdot\right)\right) \geqslant 0,
$$

then

$$
\breve{\mathrm{J}}(\mathbf{f}(\cdot)) \geqslant \breve{\mathrm{J}}\left(\mathrm{T}_{\mathrm{k}-1}\left(\mathbf{f} ; \zeta_{1}, \cdot\right)\right) ;
$$

(ii) if $\mathbf{f}$ is $\mathbf{k}$-convex function and

$$
\breve{J}\left(R_{k-1}\left(\mathbf{f} ; \zeta_{2}, \cdot\right)\right) \geqslant 0,
$$

then

$$
\breve{\mathrm{J}}(\mathbf{f}(\cdot)) \geqslant \breve{\mathrm{J}}\left(\mathrm{T}_{\mathrm{k}-1}\left(\mathbf{f} ; \zeta_{2}, \cdot\right)\right) .
$$

Proof. Since $\mathbf{f}^{(\mathrm{k}-1)}$ is absolutely continuous on $\left[\zeta_{1}, \zeta_{2}\right], \mathbf{f}^{(\mathrm{k})}$ exists almost everywhere. As $\mathbf{f}$ is $k$-convex function, therefore applying Theorem 1.2, we have, $\mathbf{f}^{(k)}(x) \geqslant 0$ for all $x \in\left[\zeta_{1}, \zeta_{2}\right]$. Hence we can apply Theorem 2.1 to obtain (2.9) and (2.11) respectively.

\section{Remark 2.3.}

(i) In Theorem 2.2, if inequalities in (2.8) and (2.10) are in reverse direction then inequalities in (2.9) and (2.11) are also reversed.

(ii) Inequalities (2.9) and (2.11) are also hold in reverse direction if the function $\mathbf{f}$ is $\mathbf{k}$-concave.

If we put $m=n, p_{\rho}=q_{\rho}$ and by using positive weights in $(2.1)$, then $\breve{\mathrm{J}}(\cdot)$ converted to the functional $\mathrm{J}(\cdot)$ defined in (1.5), also in this case, (2.2), (2.5), (2.8), (2.9), (2.10) and (2.11) become

$$
\mathrm{J}(\mathbf{f}(\cdot))=\mathrm{J}\left(\mathrm{T}_{\mathrm{k}-1}\left(\mathbf{f} ; \zeta_{1}, \cdot\right)\right)+\mathrm{J}\left(\mathrm{R}_{\mathrm{k}-1}\left(\mathbf{f} ; \zeta_{1}, \cdot\right)\right)
$$

where

$J\left(T_{k-1}\left(\mathbf{f} ; \zeta_{1}, \cdot\right)\right)=\sum_{\rho=1}^{n} p_{\rho} T_{k-1}\left(\mathbf{f} ; \zeta_{1}, y_{\rho}\right)-T_{k-1}\left(\mathbf{f} ; \zeta_{1}, \sum_{\rho=1}^{n} p_{\rho} y_{\rho}\right)-\sum_{\rho=1}^{n} p_{\rho} T_{k-1}\left(\mathbf{f} ; \zeta_{1}, x_{\rho}\right)+T_{k-1}\left(\mathbf{f} ; \zeta_{1}, \sum_{\rho=1}^{n} p_{\rho} x_{\rho}\right)$ and

$$
\begin{aligned}
J\left(R_{k-1}\left(\mathbf{f} ; \zeta_{1}, \cdot\right)\right)= & \sum_{\rho=1}^{n} p_{\rho} R_{k-1}\left(\mathbf{f} ; \zeta_{1}, y_{\rho}\right)-R_{k-1}\left(\mathbf{f} ; \zeta_{1}, \sum_{\rho=1}^{n} p_{\rho} y_{\rho}\right) \\
& -\sum_{\rho=1}^{n} p_{\rho} R_{k-1}\left(\mathbf{f} ; \zeta_{1}, x_{\rho}\right)+R_{k-1}\left(\mathbf{f} ; \zeta_{1}, \sum_{\rho=1}^{n} p_{\rho} x_{\rho}\right), \\
J(f(\cdot))= & J\left(T_{k-1}\left(\mathbf{f} ; \zeta_{2}, \cdot\right)\right)+J\left(R_{k-1}\left(\mathbf{f} ; \zeta_{2}, \cdot\right)\right),
\end{aligned}
$$

where

$$
\begin{aligned}
J\left(T_{k-1}\left(\mathbf{f} ; \zeta_{2}, \cdot\right)\right)= & \sum_{j=0}^{k-1} \frac{\mathbf{f}^{j}\left(\zeta_{2}\right)}{j !}(-1)^{j}\left(\sum_{\rho=1}^{n} p_{\rho}\left(\zeta_{2}-y_{\rho}\right)^{j}-\left(\zeta_{2}-\sum_{\rho=1}^{n} p_{\rho} y_{\rho}\right)^{j}\right. \\
& \left.-\left(\sum_{\rho=1}^{n} p_{\rho}\left(\zeta_{2}-x_{\rho}\right)^{j}\right)+\left(\zeta_{2}-\sum_{\rho=1}^{n} p_{\rho} x_{\rho}\right)^{j}\right)
\end{aligned}
$$


and

$$
\begin{aligned}
J\left(R_{k-1}\left(\mathbf{f} ; \zeta_{2}, \cdot\right)\right)= & \frac{(-1)^{k-1}}{(k-1) !} \int_{\zeta_{1}}^{\zeta_{2}}\left(\sum_{\rho=1}^{n} p_{\rho}\left(t-y_{\rho}\right)_{+}^{k-1}-\left(t-\sum_{\rho=1}^{n} p_{\rho} y_{\rho}\right)_{+}^{k-1}\right. \\
& \left.-\sum_{\rho=1}^{n} p_{\rho}\left(t-x_{\rho}\right)_{+}^{k-1}+\left(t-\sum_{\rho=1}^{n} p_{\rho} x_{\rho}\right)_{+}^{k-1}\right) \mathbf{f}^{(k)}(t) d t \\
J\left(R_{k-1}\left(\mathbf{f} ; \zeta_{1}, \cdot\right)\right) \geqslant & 0, \\
J(f(f(\cdot)) \geqslant & J\left(T_{k-1}\left(\mathbf{f} ; \zeta_{1}, \cdot\right)\right), \\
J\left(R_{k-1}\left(\mathbf{f} ; \zeta_{2}, \cdot\right)\right) \geqslant & 0 \\
J(f(\cdot)) \geqslant & J\left(T_{k-1}\left(\mathbf{f} ; \zeta_{2}, \cdot\right)\right),
\end{aligned}
$$

respectively.

Theorem 2.4. Let $\mathbf{f}^{(\mathrm{k}-1)}$ be absolutely continuous on $\mathrm{I}, \mathbf{p}=\left(\mathrm{p}_{1}, \ldots, \mathrm{p}_{\mathrm{n}}\right)$ be positive $\mathrm{n}$-tuple such that $\sum_{\rho=1}^{\mathrm{n}} \mathrm{p}_{\rho}=$ 1. Also, let $x_{\rho}, y_{\rho} \in I$ such that (1.3) is valid for $\rho=1, \ldots, n$. Then for the functional $J(\cdot)$ defined in (1.5), we have the following:

(i) if $\mathbf{f}$ is $k$-convex function, then (2.15) holds, moreover if $\mathbf{f}^{(j)}\left(\zeta_{1}\right) \geqslant 0$ for $\mathbf{j}=3, \ldots, k-1$, the R.H.S. of (2.15) will be non negative;

(ii) if $\mathrm{k}$ is even $\mathbf{f} \mathrm{k}$-convex function, then (2.17) holds, moreover if $\mathbf{f}^{(\mathbf{j})}\left(\zeta_{2}\right) \geqslant 0$ for $\mathbf{j}=4, \ldots, \mathrm{k}-2$ and $\mathbf{f}^{(\mathbf{j})}\left(\zeta_{2}\right) \leqslant 0$ for $j=3, \ldots, k-1$ the R.H.S. of (2.17) will be non negative,

(iii) if $\mathrm{k}$ is odd $\mathbf{f}$ is $\mathrm{k}$-convex function, then (2.17) holds in reverse direction, moreover if $\mathbf{f}^{(j)}\left(\zeta_{2}\right) \leqslant 0$ for $\mathbf{j}=$ $4, \ldots, k-2$ and $\mathbf{f}^{(j)}\left(\zeta_{2}\right) \geqslant 0$ for $j=3, \ldots, k-1$ the R.H.S. of $(2.17)$ will be non positive.

Proof.

(i) Since

$$
\frac{d^{3}}{d y_{\rho}^{3}}\left(y_{\rho}-t\right)_{+}^{k-1}= \begin{cases}(k-1)(k-2)(k-3)\left(y_{\rho-t}\right)^{k-4} \geqslant 0, & \text { for } t \leqslant y_{\rho}, \\ 0, & t \leqslant y_{\rho} .\end{cases}
$$

and also, $x \rightarrow(x-t)_{+}^{k-1}$ is 3-convex, therefore $(\cdot-t)_{+}^{k-1}$ is 3-convex. Since weights are positive, therefore using Remark 1.5 for $n$ points, we get (2.14). By following Theorem 2.2 (for $n=m$ and $p_{\rho}=q_{\rho}$ ), we get (2.15). Also by using Remark 1.5 and given conditions, $J\left(T_{k-1}\left(\mathbf{f} ; \zeta_{1}, \cdot\right)\right) \geqslant 0$ for $j=2, \ldots, k-1$, we get R.H.S. of (2.15) is non negative.

The pfoors of (ii) and (iii) are similar to (i).

Corollary 2.5. Let $\mathbf{f}: \mathrm{I}=\left[\zeta_{1}, \zeta_{2}\right] \rightarrow \mathbb{R}$ be such that $\mathbf{f}^{(\mathrm{k}-1)}$ is absolutely continuous. Also, let $\left(\mathrm{p}_{1}, \ldots, \mathrm{p}_{\mathrm{n}}\right)$ and $\left(\mathrm{q}_{1}, \ldots, \mathrm{q}_{\mathrm{m}}\right)$ be positive real numbers such that $\sum_{\rho=1}^{\mathfrak{n}} \mathrm{p}_{\rho}=1, \sum_{\rho=1}^{\mathrm{m}} \mathrm{q}_{\rho}=1$ and $\mathrm{x}_{\rho}, \mathrm{y}_{\rho}, \sum_{\rho=1}^{n} \mathrm{p}_{\rho} \mathrm{x}_{\rho}$, $\sum_{\rho=1}^{m} \mathrm{q}_{\rho} \mathrm{y}_{\rho} \in \mathrm{I}$. Moreover if (2.14) and (2.16) are valid then (2.15) and (2.17) are also valid, respectively.

Proof. In Theorem 2.2, choose positive real numbers such that $\sum_{\rho=1}^{n} p_{\rho}=1 \sum_{\rho=1}^{m} q_{\rho}=1$, we get required result.

Next we have generalized form (for real weights) of Levinson's type inequality for $2 n$-points given in [19] (see also [18]).

Theorem 2.6. Let $\mathbf{f}: \mathrm{I}=\left[\zeta_{1}, \zeta_{2}\right] \rightarrow \mathbb{R}$ be such that $\mathbf{f}^{(\mathrm{k}-1)}$ is absolutely continuous, $\left(\mathrm{p}_{1}, \ldots, \mathrm{p}_{\mathrm{n}}\right)$ be positive $\mathrm{n}$-tuple such that $\sum_{\rho=1}^{n} p_{\rho}=1$. Also, let $x_{\rho}, y_{\rho} \in I$ such that $x_{\rho}+y_{\rho}=2 \breve{c}, x_{\rho}+x_{n-\rho+1} \leqslant 2 \breve{c}$ and $\frac{p_{\rho} x_{\rho}+p_{n-\rho+1} x_{n-\rho+1}}{p_{\rho}+p_{n-\rho+1}} \leqslant$ $\breve{\mathrm{c}}$ for $\rho=1, \ldots, \mathrm{n}$. Then for the functional $\mathrm{J}(\cdot)$ defined in (1.5), we have the following.

(i) If $\mathbf{f}$ is $k$-convex function, then (2.15) holds. Moreover if $\mathbf{f}^{(j)}\left(\zeta_{1}\right) \geqslant 0$ for $\mathbf{j}=3, \ldots, k-1$, the R.H.S. of (2.15) will be non negative. 
(ii) If $\mathrm{k}$ is even $\mathbf{f}$ is $\mathrm{k}$-convex function, then (2.17) holds. Moreover if $\mathbf{f}^{(\mathbf{j})}\left(\zeta_{2}\right) \geqslant 0$ for $\mathbf{j}=4, \ldots, \mathrm{k}-2$ and $\mathbf{f}^{(j)}\left(\zeta_{2}\right) \leqslant 0$ for $j=3, \ldots, k-1$ the R.H.S. of (2.17) will be non negative.

(iii) If $\mathbf{k}$ is odd $\mathbf{f}$ is $\mathrm{k}$-convex function, then (2.17) holds in reverse direction. Moreover if $\mathbf{f}^{(j)}\left(\zeta_{2}\right) \leqslant 0$ for $j=4, \ldots, k-2$ and $\mathbf{f}^{(\mathfrak{j})}\left(\zeta_{2}\right) \geqslant 0$ for $j=3, \ldots, k-1$ the R.H.S. of $(2.17)$ will be non positive.

Proof. In Theorem 2.4, replace the condition (1.3), for $x_{\rho}$ and $y_{\rho}$ with the condition given in statement to get required result.

Remark 2.7. Identities (2.12) and (2.13) are also valid for the points $x_{\rho}$ and $y_{\rho}$ in Theorem 2.6 satisfying $\max \left\{x_{1} \ldots x_{n}\right\} \leqslant \min \left\{y_{1} \ldots y_{n}\right\}$ and condition (1.6).

\section{New bounds for Levinson's type inequality}

Consider the Čebyšev functional for two Lebesgue integrable functions $f_{1}, f_{2}:\left[\zeta_{1}, \zeta_{2}\right] \rightarrow \mathbb{R}$,

$$
\Theta\left(f_{1}, f_{2}\right)=\frac{1}{\zeta_{2}-\zeta_{1}} \int_{\zeta_{1}}^{\zeta_{2}} f_{1}(x) f_{2}(x) d x \frac{1}{\zeta_{2}-\zeta_{1}} \int_{\zeta_{1}}^{\zeta_{2}} f_{1}(x) d x \cdot \frac{1}{\zeta_{2}-\zeta_{1}} \int_{\zeta_{1}}^{\zeta_{2}} f_{2}(x) d x,
$$

where the integrals are assumed to exist.

Theorem 3.1 ([9]). Let $\mathrm{f}_{1}:\left[\zeta_{1}, \zeta_{2}\right] \rightarrow \mathbb{R}$ be a Lebesgue integrable function and $\mathrm{f}_{2}:\left[\zeta_{1}, \zeta_{2}\right] \rightarrow \mathbb{R}$ be an absolutely continuous function with $\left(.,-\zeta_{1}\right)\left(.,-\zeta_{2}\right)\left[f_{2}^{\prime}\right]^{2} \in L\left[\zeta_{1}, \zeta_{2}\right]$. Then

$$
\left|\Theta\left(f_{1}, f_{2}\right)\right| \leqslant \frac{1}{\sqrt{2}}\left[\Theta\left(f_{1}, f_{1}\right)\right]^{\frac{1}{2}} \frac{1}{\sqrt{\zeta_{2}-\zeta_{1}}}\left(\int_{\zeta_{1}}^{\zeta_{2}}\left(t-\zeta_{1}\right)\left(\zeta_{2}-t\right)\left[f_{2}^{\prime}(t)\right]^{2} d t\right)^{\frac{1}{2}},
$$

$\frac{1}{\sqrt{2}}$ is the best possible.

Theorem 3.2 ([9]). Let $\mathrm{f}_{1}:\left[\zeta_{1}, \zeta_{2}\right] \rightarrow \mathbb{R}$ be an absolutely continuous with $\mathrm{f}_{1}^{\prime} \in \mathrm{L}_{\infty}\left[\zeta_{1}, \zeta_{2}\right]$ and $\mathrm{f}_{2}:\left[\zeta_{1}, \zeta_{2}\right] \rightarrow \mathbb{R}$ is monotonic non-decreasing on $\left[\zeta_{1}, \zeta_{2}\right]$. Then

$$
\left|\Theta\left(f_{1}, f_{2}\right)\right| \leqslant \frac{1}{2\left(\zeta_{2}-\zeta_{1}\right)}\left\|f^{\prime}\right\|_{\infty} \int_{\zeta_{1}}^{\zeta_{2}}\left(t-\zeta_{1}\right)\left(\zeta_{2}-t\right)\left[f_{2}^{\prime}(t)\right]^{2} d f_{2}(t),
$$

$\frac{1}{2}$ is the best possible.

To generalize results given in previous section we will consider Theorems 3.1 and 3.2.

Theorem 3.3. Let $\mathbf{f}: \mathrm{I}=\left[\zeta_{1}, \zeta_{2}\right] \rightarrow \mathbb{R}$ be such that $\mathbf{f}^{(\mathrm{k})}(\cdot)$ is absolutely continuous with $\left(.-\zeta_{1}\right)\left(\zeta_{2}-.\right)\left[\mathbf{f}^{(\mathrm{k}+1)}\right]^{2} \in$ $\mathrm{L}\left[\zeta_{1}, \zeta_{2}\right]$. Also, let $\left(\mathrm{p}_{1}, \ldots, \mathrm{p}_{\mathrm{n}}\right) \in \mathbb{R}^{\mathrm{n}},\left(\mathrm{q}_{1}, \ldots, \mathrm{q}_{\mathrm{m}}\right) \in \mathbb{R}^{\mathrm{m}}$ be such that $\sum_{\rho=1}^{\mathrm{n}} \mathrm{p}_{\rho}=1, \sum_{\rho=1}^{\mathrm{m}} \mathrm{q}_{\rho}=1$, $x_{\rho}, y_{\rho}, \sum_{\rho=1}^{n} p_{\rho} x_{\rho}, \sum_{\rho=1}^{m} q_{\rho} y_{\rho} \in I$. Then

(i)

$$
\breve{J}(\mathbf{f}(\cdot))=\breve{J}\left(\mathrm{~T}_{\mathrm{k}-1}\left(\mathbf{f} ; \zeta_{1}, \cdot\right)\right)+\frac{\mathbf{f}^{(\mathrm{k}-1)}\left(\zeta_{2}\right)-\mathbf{f}^{(\mathrm{k}-1)}\left(\zeta_{1}\right)}{\left(\zeta_{2}-\zeta_{1}\right)} \breve{\mathrm{J}}\left(\mathrm{R}_{\mathrm{k}-1}\left(\mathbf{f} ; \zeta_{1}, \cdot\right)\right)+\mathcal{R}_{\mathrm{m}}\left(\zeta_{1}, \zeta_{2} ; \mathbf{f}\right),
$$

where $\breve{J}(\mathbf{f}(\cdot)), \breve{J}\left(T_{k-1}\left(\mathbf{f} ; \zeta_{1}, \cdot\right)\right)$ and $\breve{J}\left(R_{k-1}\left(\mathbf{f} ; \zeta_{1}, \cdot\right)\right)$ are defined in (2.1), (2.3), and (2.4), respectively, where the remainder $\mathcal{R}_{k}\left(\zeta_{1}, \zeta_{2} ; \mathbf{f}\right)$ satisfies the bound

$$
\begin{aligned}
\left|\mathcal{R}_{k}\left(\zeta_{1}, \zeta_{2} ; \mathbf{f}\right)\right| \leqslant & \frac{\left(\zeta_{2}-\zeta_{1}\right)}{\sqrt{2}}\left[\Theta\left(\breve{J}\left(R_{k-1}\left(\mathbf{f} ; \zeta_{1}, \cdot\right)\right), \breve{J}\left(R_{k-1}\left(\mathbf{f} ; \zeta_{1}, \cdot\right)\right)\right)\right]^{\frac{1}{2}} \\
& \times \frac{1}{\sqrt{\zeta_{2}-\zeta_{1}}}\left(\int_{\zeta_{1}}^{\zeta_{2}}\left(t-\zeta_{1}\right)\left(\zeta_{2}-t\right)\left[\mathbf{f}^{(k+1)}(t)\right]^{2} d t\right)^{\frac{1}{2}} .
\end{aligned}
$$


(ii)

$$
\breve{J}(\mathbf{f}(\cdot))=\breve{J}\left(T_{k-1}\left(\mathbf{f} ; \zeta_{2}, \cdot\right)\right)+\frac{\mathbf{f}^{(k-1)}\left(\zeta_{2}\right)-\mathbf{f}^{(k-1)}\left(\zeta_{1}\right)}{\left(\zeta_{2}-\zeta_{1}\right)} \breve{J}\left(R_{k-1}\left(\mathbf{f} ; \zeta_{2}, \cdot\right)\right)+\mathcal{R}_{m}^{\prime}\left(\zeta_{1}, \zeta_{2} ; \mathbf{f}\right),
$$

where $\breve{\mathrm{J}}(\mathbf{f}(\cdot)), \breve{\mathrm{J}}\left(\mathrm{T}_{\mathrm{k}-1}\left(\mathbf{f} ; \zeta_{2}, \cdot\right)\right)$ and $\breve{\mathrm{J}}\left(\mathrm{R}_{\mathrm{k}-1}\left(\mathbf{f} ; \zeta_{2}, \cdot\right)\right)$ are defined in $(2.1),(2.6)$, and (2.7), respectively, where the remainder $\mathcal{R}_{k}\left(\zeta_{1}, \zeta_{2} ; \mathbf{f}\right)$ satisfies the bound

$$
\begin{aligned}
\left|\mathcal{R}_{\mathrm{k}}^{\prime}\left(\zeta_{1}, \zeta_{2} ; \mathbf{f}\right)\right| \leqslant & \frac{\left(\zeta_{2}-\zeta_{1}\right)}{\sqrt{2}}\left[\Theta\left(\breve{J}\left(R_{\mathrm{k}-1}\left(\mathbf{f} ; \zeta_{1}, \cdot\right)\right), \breve{J}\left(R_{k-1}\left(\mathbf{f} ; \zeta_{1}, \cdot\right)\right)\right)\right]^{\frac{1}{2}} \\
& \times \frac{1}{\sqrt{\zeta_{2}-\zeta_{1}}}\left(\int_{\zeta_{1}}^{\zeta_{2}}\left(t-\zeta_{1}\right)\left(\zeta_{2}-t\right)\left[\mathbf{f}^{(k+1)}(t)\right]^{2} d t\right)^{\frac{1}{2}} .
\end{aligned}
$$

Proof.

(i) Setting $f_{1} \mapsto \breve{J}\left(R_{k-1}\left(\mathbf{f} ; \zeta_{1}, \cdot\right)\right)$ and $f_{2} \mapsto \mathbf{f}^{(k)}$ in Theorem 3.1, we get

$$
\begin{aligned}
& \left|\frac{1}{\zeta_{2}-\zeta_{1}} \int_{\zeta_{1}}^{\zeta_{2}} \breve{J}\left(R_{k-1}\left(\mathbf{f} ; \zeta_{1}, \cdot\right)\right) \mathbf{f}^{(k)}(t) d t-\frac{1}{\zeta_{2}-\zeta_{1}} \int_{\zeta_{1}}^{\zeta_{2}} \breve{J}\left(R_{k-1}\left(\mathbf{f} ; \zeta_{1}, \cdot\right)\right) d t \frac{1}{\zeta_{2}-\zeta_{1}} \int_{\zeta_{1}}^{\zeta_{2}} \mathbf{f}^{(k)}(s) d t\right| \\
& \quad \leqslant \frac{1}{\sqrt{2}}\left[\Theta\left(\breve{J}\left(R_{k-1}\left(\mathbf{f} ; \zeta_{1}, \cdot\right)\right), \breve{J}\left(R_{k-1}\left(\mathbf{f} ; \zeta_{1}, \cdot\right)\right)\right)\right]^{\frac{1}{2}} \frac{1}{\sqrt{\zeta_{2}-\zeta_{1}}}\left(\int_{\zeta_{1}}^{\zeta_{2}}\left(t-\zeta_{1}\right)\left(\zeta_{2}-t\right)\left[\mathbf{f}^{(k+1)}(t)\right]^{2} d t\right)^{\frac{1}{2}}, \\
& \left|\frac{1}{\zeta_{2}-\zeta_{1}} \int_{\zeta_{1}}^{\zeta_{2}} \breve{J}\left(R_{k-1}\left(\mathbf{f} ; \zeta_{1}, \cdot\right)\right) \mathbf{f}^{(k)}(s) d t-\frac{f^{(2)}\left(\zeta_{2}\right)-\mathbf{f}^{(2)}\left(\zeta_{1}\right)}{\left(\zeta_{2}-\zeta_{1}\right)^{2}} \int_{\zeta_{1}}^{\zeta_{2}} \breve{J}\left(R_{k-1}\left(\mathbf{f} ; \zeta_{1}, \cdot\right)\right) d t\right| \\
& \quad \leqslant \frac{1}{\sqrt{2}}\left[\Theta\left(\breve{J}\left(R_{k-1}\left(\mathbf{f} ; \zeta_{1}, \cdot\right)\right), \breve{J}\left(R_{k-1}\left(\mathbf{f} ; \zeta_{1}, \cdot\right)\right)\right]^{\frac{1}{2}} \frac{1}{\sqrt{\zeta_{2}-\zeta_{1}}}\left(\int_{\zeta_{1}}^{\zeta_{2}}\left(t-\zeta_{1}\right)\left(\zeta_{2}-t\right)\left[\mathbf{f}^{(k+1)}(s)\right]^{2} d t\right)^{\frac{1}{2}} .\right.
\end{aligned}
$$

Multiplying $\left(\zeta_{2}-\zeta_{1}\right)$ on both sides of above inequality and using the estimation (3.2), we get

$$
\int_{\zeta_{1}}^{\zeta_{2}} \breve{J}\left(R_{k-1}\left(\mathbf{f} ; \zeta_{1}, \cdot\right)\right) \mathbf{f}^{(k)} d t=\frac{f^{(2)}\left(\zeta_{2}\right)-\mathbf{f}^{(2)}\left(\zeta_{1}\right)}{\left(\zeta_{2}-\zeta_{1}\right)} \int_{\zeta_{1}}^{\zeta_{2}} \breve{J}\left(R_{k-1}\left(\mathbf{f} ; \zeta_{1}, \cdot\right)\right) d t+\mathcal{R}_{k}\left(\zeta_{1}, \zeta_{1} ; \mathbf{f}\right) .
$$

Using identity (2.1), we get (3.1).

(ii) Similar to above part.

By using Theorem 3.2, Grüss type inequalities can be obtained.

Theorem 3.4. Let $\mathbf{f}: \mathrm{I}=\left[\zeta_{1}, \zeta_{2}\right] \rightarrow \mathbb{R}$ be such that $\mathbf{f}^{(\mathrm{k})}(\cdot)$ is absolutely continuous and $\mathbf{f}^{(\mathrm{k}+1)}(\cdot) \geqslant 0$ a.e. on $\left[\zeta_{1}, \zeta_{2}\right]$. Also, let $\left(\mathrm{p}_{1}, \ldots, \mathrm{p}_{\mathrm{n}}\right) \in \mathbb{R}^{\mathrm{n}},\left(\mathrm{q}_{1}, \ldots, \mathrm{q}_{\mathrm{m}}\right) \in \mathbb{R}^{\mathrm{m}}$ be such that $\sum_{\rho=1}^{\mathrm{n}} \mathrm{p}_{\rho}=1, \sum_{\rho=1}^{\mathrm{m}} \mathrm{q}_{\rho}=1$, $x_{\rho}, y_{\rho}, \sum_{\rho=1}^{n} p_{\rho} x_{\rho}, \sum_{\rho=1}^{m} q_{\rho} y_{\rho} \in I$. Then

(i) Identity (3.1) holds, where the remainder satisfies the estimation

$$
\left|\mathcal{R}_{k}\left(\zeta_{1}, \zeta_{2} ; \mathbf{f}\right)\right| \leqslant\left(\zeta_{2}-\zeta_{1}\right)\left\|\left(\breve{J}\left(R_{k-1}\left(\mathbf{f} ; \zeta_{1}, \cdot\right)\right)\right)^{\prime}\right\|_{\infty}\left[\frac{\mathbf{f}^{(k-1)}\left(\zeta_{2}\right)+\mathbf{f}^{(k-1)}\left(\zeta_{1}\right)}{2}-\frac{\mathbf{f}^{(k-2)}\left(\zeta_{2}\right)-\mathbf{f}^{(k-2)}\left(\zeta_{1}\right)}{\zeta_{2}-\zeta_{1}}\right] .
$$

(ii) Identity (3.3) holds, where the remainder satisfies the estimation

$$
\left.\left|\mathcal{R}_{k}^{\prime}\left(\zeta_{1}, \zeta_{2} ; \mathbf{f}\right)\right| \leqslant\left(\zeta_{2}-\zeta_{1}\right) \|\left(\breve{J}\left(R_{k-1}\left(\mathbf{f} ; \zeta_{1}, \cdot\right)\right)\right)\right)^{\prime} \|_{\infty}\left[\frac{\mathbf{f}^{(k-1)}\left(\zeta_{2}\right)+\mathbf{f}^{(k-1)}\left(\zeta_{1}\right)}{2}-\frac{\mathbf{f}^{(k-2)}\left(\zeta_{2}\right)-\mathbf{f}^{(k-2)}\left(\zeta_{1}\right)}{\zeta_{2}-\zeta_{1}}\right] .
$$


Proof.

(i) Setting $f_{1} \mapsto \breve{J}\left(R_{k-1}\left(f ; \zeta_{1},.\right)\right)$ and $f_{2} \mapsto \mathbf{f}^{(k)}$ in Theorem 3.2, we get

$$
\begin{aligned}
& \left|\frac{1}{\zeta_{2}-\zeta_{1}} \int_{\zeta_{1}}^{\zeta_{2}} \breve{J}\left(R_{k-1}\left(\mathbf{f} ; \zeta_{1}, \cdot\right)\right) \mathbf{f}^{(k)}(t) d t-\frac{1}{\zeta_{2}-\zeta_{1}} \int_{\zeta_{1}}^{\zeta_{2}} \breve{J}\left(R_{k-1}\left(\mathbf{f} ; \zeta_{1}, \cdot\right)\right) d t \frac{1}{\zeta_{2}-\zeta_{1}} \int_{\zeta_{1}}^{\zeta_{2}} \mathbf{f}^{(k)}(t) d t\right| \\
& \leqslant \frac{1}{2}\left\|\left(\breve{J}\left(R_{k-1}\left(\mathbf{f} ; \zeta_{1}, \cdot\right)\right)\right)^{\prime}\right\|_{\infty} \frac{1}{\zeta_{2}-\zeta_{1}} \int_{\zeta_{1}}^{\zeta_{2}}\left(t-\zeta_{1}\right)\left(\zeta_{2}-t\right)\left[\mathbf{f}^{(k+1)}(t)\right]^{2} d t .
\end{aligned}
$$

Since,

$$
\begin{aligned}
\int_{\zeta_{1}}^{\zeta_{2}}\left(t-\zeta_{1}\right)\left(\zeta_{2}-t\right)\left[f^{(k+1)}(t)\right]^{2} d t & =\int_{\zeta_{1}}^{\zeta_{2}}\left[2 t-\zeta_{1}-\zeta_{2}\right] \mathbf{f}^{k}(t) d t \\
& =\left(\zeta_{2}-\zeta_{1}\right)\left[\mathbf{f}^{(k-1)}\left(\zeta_{2}\right)+\mathbf{f}^{(k-1)}\left(\zeta_{1}\right)\right]-2\left(\mathbf{f}^{(k-1)}\left(\zeta_{2}\right)-\mathbf{f}^{(k-1)}\left(\zeta_{1}\right)\right)
\end{aligned}
$$

using (2.1), (3.4) and (3.5), we have (3.1).

(ii) Similar to above part.

Theorem 3.5. Let $\mathbf{f}: \mathrm{I}=\left[\zeta_{1}, \zeta_{2}\right] \rightarrow \mathbb{R}$ be such that $\mathbf{f}^{(\mathrm{k}-1)}(\cdot)$ is absolutely continuous. Also, let $\left(\mathrm{p}_{1}, \ldots, \mathrm{p}_{\mathrm{n}}\right) \in \mathbb{R}^{\mathrm{n}}$, $\left(q_{1}, \ldots, q_{m}\right) \in \mathbb{R}^{m}$ be such that $\sum_{\rho=1}^{n} p_{\rho}=1, \sum_{\rho=1}^{m} q_{\rho}=1, x_{\rho}, y_{\rho}, \sum_{\rho=1}^{n} p_{\rho} x_{\rho}, \sum_{\rho=1}^{m} q_{\rho} y_{\rho} \in I$. Also, let $(\mathrm{r}, \mathrm{s})$ be a pair of conjugate exponents that is $1 \leqslant \mathrm{r}, \mathrm{s}, \leqslant \infty, \frac{1}{\mathrm{r}}+\frac{1}{\mathrm{~s}}=1$. If $\left|\mathbf{f}^{(\mathrm{k})}\right|^{\mathrm{r}}:\left[\zeta_{1}, \zeta_{2}\right] \rightarrow \mathbb{R}$ be Riemann integrable function, then

(i)

$$
\left|\breve{J}(\mathbf{f}(\cdot))-\breve{J}\left(T_{k-1}\left(\mathbf{f} ; \zeta_{1}, \cdot\right)\right)\right| \leqslant\left\|\mathbf{f}^{(k)}\right\|_{\mathrm{r}}\left(\int_{\zeta_{1}}^{\zeta_{2}}\left|\breve{J}\left(R_{k-1}\left(\mathbf{f} ; \zeta_{1}, \cdot\right)\right) d t\right|^{s}\right)^{\frac{1}{s}} ;
$$

(ii)

$$
\left|\breve{J}(\mathbf{f}(\cdot))-\breve{J}^{\prime}\left(T_{k-1}\left(\mathbf{f} ; \zeta_{2}, \cdot\right)\right)\right| \leqslant\left\|\mathbf{f}^{(k)}\right\|_{\mathbf{r}}\left(\int_{\zeta_{1}}^{\zeta_{2}}\left|\breve{J}^{\prime}\left(R_{k-1}\left(\mathbf{f} ; \zeta_{2}, \cdot\right)\right) d t\right|^{s}\right)^{\frac{1}{s}} .
$$

Proof. For proof see Theorem 3.5 in [8].

Remark 3.6. Similar work can be done for Levinson's inequality (1.2) (one type of data points) for higher order-convex functions.

Remark 3.7. We can give related mean value theorems by using non-negative functionals (2.2) and (2.5) and also, we can construct the new families of k-exponentially convex functions and Cauchy means related to these functionals as given in Section 4 of [8].

\section{Application to information theory}

The idea of Shannon entropy is the central job of information speculation, now and again implied as measure of uncertainty. The entropy of a random variable is described with respect to probability distribution, and it can be shown that it is a decent measure of random. The assignment of Shannon entropy is to assess the typical least number of bits expected to encode a progression of pictures subject to the letters, including the size and the repetition of the symbols.

Divergences between probability distributions can be interpreted as measure of the distance between them. An assortment of sorts of divergences exist, for example the f-divergences (especially, KullbackLeibler divergences, Hellinger distance and total variation distance), Rényi divergences, Jensen-Shannon divergences, etc (see $[16,25])$. There are a lot of papers dealing with the subject of inequalities and entropies, see, e.g., $[1-5,12,14,24]$ and the references therein. Jensen's inequality deals with one kind of data points, Levinson's inequality deals two types of data points. 


\subsection{Csiszár divergence}

Following definition is given by Csiszár in $[10,11]$.

Definition 4.1. Let $\mathbf{f}: \mathbb{R}^{+} \rightarrow \mathbb{R}^{+}$be a convex function. Also, let $\tilde{\mathbf{r}}, \tilde{\mathbf{k}} \in \mathbb{R}_{+}^{n}$ be such that $\sum_{v=1}^{n} \mathrm{r}_{v}=1$ and $\sum_{v=1}^{n} k_{v}=1$. Then $\mathbf{f}$-divergence functional is defined by

$$
\mathrm{I}_{\mathbf{f}}(\tilde{\mathbf{r}}, \tilde{\mathbf{k}}):=\sum_{v=1}^{\mathrm{n}} \mathrm{k}_{v} \mathrm{f}\left(\frac{\mathrm{r}_{v}}{\mathrm{k}_{v}}\right) .
$$

Define the following:

$$
\mathbf{f}(0):=\lim _{x \rightarrow 0^{+}} \mathbf{f}(x) ; \quad \text { of }\left(\frac{0}{0}\right):=0 ; \quad \text { of }\left(\frac{a}{0}\right):=\lim _{x \rightarrow 0^{+}} x \mathbf{f}\left(\frac{a}{x}\right), \quad a>0 .
$$

Definition 4.2 ([13]). Let I be an interval contained in $\mathbb{R}$ and $\mathbf{f}: \mathrm{I} \rightarrow \mathbb{R}$ be a function. Also, let $\tilde{\mathbf{r}}=$ $\left(r_{1}, \ldots, r_{n}\right) \in \mathbb{R}^{\mathfrak{n}}$ and $\tilde{\mathbf{k}}=\left(k_{1}, \ldots, k_{n}\right) \in(0, \infty)^{\mathfrak{n}}$ be such that

$$
\frac{\mathrm{r}_{v}}{\mathrm{k}_{v}} \in \mathrm{I}, \quad v=1, \ldots, \mathrm{n}
$$

Then

$$
\hat{\mathrm{I}}_{\mathbf{f}}(\tilde{\mathbf{r}}, \tilde{\mathbf{k}}):=\sum_{v=1}^{\mathrm{n}} \mathrm{k}_{v} \mathbf{f}\left(\frac{\mathrm{r}_{v}}{\mathrm{k}_{v}}\right)
$$

We apply Theorem 2.2 for k-convex functions to $\hat{\mathrm{I}}_{\mathbf{f}}(\tilde{\mathbf{r}}, \tilde{\mathbf{k}})$.

Theorem 4.3. Let $\tilde{\mathbf{r}}=\left(r_{1}, \ldots, r_{n}\right) \in \mathbb{R}^{n}, \tilde{\mathbf{w}}=\left(w_{1}, \ldots, w_{m}\right) \in \mathbb{R}^{m}, \tilde{\mathbf{k}}=\left(k_{1}, \ldots, k_{n}\right) \in(0, \infty)^{n}$ and $\tilde{\mathbf{t}}=$ $\left(\mathrm{t}_{1}, \ldots, \mathrm{t}_{\mathrm{m}}\right) \in(0, \infty)^{\mathrm{m}}$ be such that

$$
\frac{\mathrm{r}_{v}}{\mathrm{k}_{v}} \in \mathrm{I}, \quad v=1, \ldots, \mathrm{n}, \quad \text { and } \quad \frac{w_{\mathrm{u}}}{\mathrm{t}_{\mathrm{u}}} \in \mathrm{I}, \quad \mathrm{u}=1, \ldots, \mathrm{m}
$$

Also, let $\mathbf{f}: \mathrm{I}=\left[\zeta_{1}, \zeta_{2}\right] \rightarrow \mathbb{R}$ be such that $\mathbf{f}^{\mathrm{k}-1}$ is absolutely continuous and $\mathbf{f}$ is $\mathrm{k}$-convex function, then

(i)

$$
\mathrm{J}_{\mathrm{cis}}(\mathbf{f}(\cdot)) \geqslant \mathrm{J}\left(\mathrm{T}_{\mathrm{k}-1}\left(\mathbf{f} ; \zeta_{1}, \cdot\right)\right)
$$

where

$$
\mathrm{J}_{\text {cis }}(\mathbf{f}(\cdot))=\frac{1}{\sum_{\mathfrak{u}=1}^{m} \mathrm{t}_{\mathfrak{u}}} \hat{\mathrm{I}}_{\mathbf{f}}(\tilde{\mathbf{w}}, \tilde{\mathbf{t}})-\mathbf{f}\left(\sum_{\mathfrak{u}=1}^{\mathrm{m}} \frac{w_{\mathfrak{u}}}{\sum_{\mathfrak{u}=1}^{m} \mathrm{t}_{\mathfrak{u}}}\right)-\frac{1}{\sum_{v=1}^{n} \mathrm{k}_{v}} \hat{\mathrm{I}}_{\mathbf{f}}(\tilde{\mathbf{r}}, \tilde{\mathbf{k}})+\mathbf{f}\left(\sum_{v=1}^{n} \frac{\mathrm{r}_{v}}{\sum_{v=1}^{n} \mathrm{k}_{v}}\right)
$$

and

$$
\begin{aligned}
J\left(T_{k-1}\left(\mathbf{f} ; \zeta_{1}, \cdot\right)\right)= & \sum_{\rho=1}^{m} \frac{t_{\mathfrak{u}}}{\sum_{\mathfrak{u}=1}^{m} t_{\mathfrak{u}}} \mathrm{T}_{k-1}\left(\mathbf{f} ; \zeta_{1}, \frac{w_{\mathfrak{u}}}{\mathrm{t}_{\mathfrak{u}}}\right)-\mathrm{T}_{\mathrm{k}-1}\left(\mathbf{f} ; \zeta_{1}, \sum_{\rho=1}^{\mathrm{m}} \frac{w_{\mathfrak{u}}}{\sum_{\mathfrak{u}=1}^{m} \mathrm{t}_{\mathfrak{u}}}\right) \\
& -\sum_{\rho=1}^{n} \frac{k_{v}}{\sum_{v=1}^{n} k_{v}} \mathrm{~T}_{k-1}\left(\mathbf{f} ; \zeta_{1}, \frac{\mathrm{r}_{v}}{k_{v}}\right)+\mathrm{T}_{k-1}\left(\mathbf{f} ; \zeta_{1}, \sum_{\rho=1}^{n} \frac{r_{v}}{\sum_{v=1}^{n} k_{v}}\right) ;
\end{aligned}
$$


(ii)

$$
\mathrm{J}_{\mathrm{cis}}(\mathbf{f}(\cdot)) \geqslant \mathrm{J}\left(\mathrm{T}_{\mathrm{k}-1}\left(\mathbf{f} ; \zeta_{2}, \cdot\right)\right)
$$

where

$$
\begin{aligned}
J\left(T_{k-1}\left(\mathbf{f} ; \zeta_{2}, \cdot\right)\right)= & \sum_{j=0}^{k-1} \frac{\mathbf{f}^{\mathfrak{j}}\left(\zeta_{2}\right)}{j !}(-1)^{j}\left[\sum_{\rho=1}^{m} \frac{t_{u}}{\sum_{u=1}^{m} t_{\mathfrak{u}}}\left(\zeta_{2}-\frac{w_{\mathfrak{u}}}{t_{\mathfrak{u}}}\right)^{j}-\left(\zeta_{2}-\sum_{\rho=1}^{m} \frac{w_{\mathfrak{u}}}{\sum_{u=1}^{m} t_{\mathfrak{u}}}\right)^{j}\right. \\
& \left.-\left(\sum_{\rho=1}^{n} \frac{k_{v}}{\sum_{v=1}^{n} k_{v}}\left(\zeta_{2}-\frac{r_{v}}{k_{v}}\right)^{j}\right)+\left(\zeta_{2}-\sum_{\rho=1}^{n} \frac{r_{v}}{\sum_{v=1}^{n} k_{v}}\right)^{j}\right] .
\end{aligned}
$$

Proof.

(i) Since $\mathbf{f}$ is k-convex and

$$
\frac{d^{3}}{d(\cdot)^{3}}(\cdot-t)_{+}^{k-1}= \begin{cases}(k-1)(k-2)(k-3)(\cdot-t)^{k-4} \geqslant 0, & \text { for } t \leqslant \cdot \\ 0, & t \leqslant \cdot\end{cases}
$$

hence $(\cdot-t)_{+}^{k-1}$ is 3-convex, therefore (2.8) holds. Using $p_{\rho}=\frac{k_{v}}{\sum_{v=1}^{n} k_{v}}, x_{\rho}=\frac{r_{v}}{k_{v}}, q_{\rho}=\frac{t_{u}}{\sum_{u=1}^{m} t_{u}}, y_{\rho}=\frac{w_{u}}{t_{u}}$ in Theorem $2.2(\mathbf{i}),(2.9)$ becomes (4.2), where $\hat{I}_{\mathbf{f}}(\tilde{\mathbf{r}}, \tilde{\mathbf{k}})$ is defined in (4.1) and

$$
\hat{\mathrm{I}}_{\mathbf{f}}(\tilde{\mathbf{w}}, \tilde{\mathbf{t}}):=\sum_{\mathfrak{u}=1}^{m} t_{\mathfrak{u}} \mathbf{f}\left(\frac{w_{\mathfrak{u}}}{t_{\mathfrak{u}}}\right)
$$

(ii) By using the same substitutions as in Theorem 2.2 (ii) and following the similar steps as in (i), (2.11) becomes (4.3).

\subsection{Shannon Entropy}

Definition 4.4 ([13]). The Shannon entropy of positive probability distribution $\tilde{\mathbf{k}}=\left(\mathrm{k}_{1}, \ldots, \mathrm{k}_{\mathrm{n}}\right)$ is defined by

$$
\mathcal{S}:=-\sum_{v=1}^{n} k_{v} \log \left(k_{v}\right)
$$

Corollary 4.5. Let $\tilde{\mathbf{k}}=\left(\mathrm{k}_{1}, \ldots, \mathrm{k}_{\mathrm{n}}\right)$ and $\tilde{\mathbf{t}}=\left(\mathrm{t}_{1}, \ldots, \mathrm{t}_{\mathrm{m}}\right)$ be positive probability distributions. Also, let $\tilde{\mathbf{r}}=$ $\left(\mathrm{r}_{1}, \ldots, \mathrm{r}_{\mathrm{n}}\right) \in(0, \infty)^{\mathrm{n}}$ and $\tilde{\mathbf{w}}=\left(w_{1}, \ldots, w_{\mathrm{m}}\right) \in(0, \infty)^{\mathrm{m}}$. If base of $\log$ is greater than 1 and $\mathrm{k}=$ odd $(\mathrm{k}=3,5, \ldots)$, then

(i)

$$
\mathrm{J}_{\mathrm{s}}(\cdot) \geqslant \mathrm{J}\left(\mathrm{T}_{\mathrm{k}-1}\left(\log \mathrm{x} ; \zeta_{1}, \cdot\right)\right)
$$

where

$$
\mathrm{T}_{k-1}\left(\log x ; \zeta_{1}, \cdot\right)=\sum_{j=0}^{k-1} \frac{(-1)^{j-1}(j-1) !}{j !\left(\zeta_{1}\right)^{j}}(\cdot-c)^{j}
$$

and

$$
J_{s}(\cdot)=\sum_{\mathfrak{u}=1}^{m} t_{\mathfrak{u}} \log \left(w_{\mathfrak{u}}\right)+\tilde{\mathcal{S}}-\log \left(\sum_{\mathfrak{u}=1}^{\mathrm{m}} w_{\mathfrak{u}}\right)-\sum_{v=1}^{n} k_{v} \log \left(r_{v}\right)+\mathcal{S}+\log \left(\sum_{v=1}^{n} r_{v}\right) .
$$


(ii)

$$
\mathrm{J}_{\mathrm{s}}(\cdot) \geqslant \mathrm{J}\left(\mathrm{T}_{\mathrm{k}-1}\left(\log \mathrm{x} ; \zeta_{2}, \cdot\right)\right)
$$

where

$$
\begin{aligned}
J\left(T_{k-1}\left(\log x ; \zeta_{2}, \cdot\right)\right)= & \sum_{j=0}^{k-1} \frac{(-1)^{j}(j-1) !}{j !\left(\zeta_{2}\right)^{j}}\left(\sum_{\rho=1}^{m} \frac{t_{\mathfrak{u}}}{\sum_{\mathfrak{u}=1}^{m} t_{\mathfrak{u}}}\left(\zeta_{2}-\frac{w_{\mathfrak{u}}}{t_{\mathfrak{u}}}\right)^{j}\right. \\
& \left.-\left(\zeta_{2}-\sum_{\rho=1}^{m} \frac{w_{\mathfrak{u}}}{\sum_{\mathfrak{u}=1}^{m} t_{\mathfrak{u}}}\right)^{j}-\left(\sum_{\rho=1}^{n} \frac{k_{v}}{\sum_{v=1}^{n} k_{v}}\left(\zeta_{2}-\frac{r_{v}}{k_{v}}\right)^{j}\right)+\left(\zeta_{2}-\sum_{\rho=1}^{n} \frac{r_{v}}{\sum_{v=1}^{n} k_{v}}\right)^{j}\right) ;
\end{aligned}
$$

(iii) if base of $\log$ is less than 1 or $k=e v e n(k=4,6, \ldots)$, then inequalities (4.5) and (4.7) are hold in reverse direction.

Proof.

(i) The function $\mathbf{f} \rightarrow \log (x)$ is $k$-convex for $k=3,5, \ldots$ and base of $\log$ is greater than 1 . Therefore using $\mathbf{f}=\log (x)$ in Theorem 4.3 (i), we get (4.5), where $\mathcal{S}$ is defined in (4.4) and

$$
\tilde{\mathcal{S}}=-\sum_{\mathfrak{u}=1}^{m} t_{\mathfrak{u}} \log \left(t_{\mathfrak{u}}\right) .
$$

(ii) Using $\mathbf{f}=\log (x)$ in Theorem 4.3 (ii), we get (4.7).

(iii) Since the function $\mathbf{f} \rightarrow \log (\mathrm{x})$ is $k$-concave for $k=4,6, \ldots$, so by using Remark 2.3 (ii), (2.9) and (2.11) are hold in reverse direction. Therefore using $\mathbf{f}=\log (x)$ and $p_{\rho}=\frac{k_{v}}{\sum_{v=1}^{n} k_{v}}, x_{\rho}=\frac{r_{v}}{k_{v}}, q_{\rho}=\frac{t_{u}}{\sum_{u=1}^{\frac{m}{m}} t_{u}}, y_{\rho}=\frac{w_{u}}{t_{u}}$ in reversed inequalities of (2.9) and (2.11), we get (4.5) and (4.7) in reverse directions respectively.

\section{Acknowledgment}

The authors wish to thanks the anonymous referees for their very careful reading of the manuscript and fruitful comments and suggestions. The research of the 4rth author is supported by the Ministry of Education and Science of the Russian Federation (the Agreement number No. 02.a03.21.0008).

\section{References}

[1] M. Adeel, K. A. Khan, Đ. Pečarić, J. Pečarić, Generalization of the Levinson inequality with applications to information theorey, J. Inequal. Appl., 2019 (2019), 19 pages. 4

[2] M. Adeel, K. A. Khan, Đ. Pečarić, J. Pečarić, Levinson type inequalities for higher order convex functions via AbelGontscharoff interpolation, Adv. Difference Equ., 2019 (2019), 13 pages.

[3] M. Adeel, K. A. Khan, Đ. Pečarić, J. Pečarić, Estimation of f-divergence and Shannon entropy by Levinson type inequalities via new Green's functions and Lidstone polynomial, Adv. Difference Equ., 2020 (2020), 15 pages.

[4] M. Adeel, K. A. Khan, Đ. Pečarić, J. Pečarić, Estimation of f-divergence and Shannon entropy by Levinson type inequalities via Fink's identity, Accepted in Filomat.

[5] M. Adeel, K. A. Khan, Đ. Pečarić, J. Pečarić, Levinson-type Inequalities via new Green functions and Montgomery identity, Accepted in Open Mathematics. 4

[6] R. P. Agarwal, P. J. Y. Wong, Error Inequalities in Polynomial Interpolation and Their Applications, Kluwer Academic Publ., Dordrecht, (1983). 1

[7] P. S. Bullen, An inequality of N. Levinson, Univ. Beograd. Publ. Elektrotehn. Fak. Ser. Mat. Fiz., $412 / 460$ (1973), 109-112. 1

[8] S. I. Butt, K. A. Khan, J. Pečarić, Generalization of Popoviciu inequality for higher order convex function via Taylor's polynomial, Acta Univ. Apulensis Math. Inform., 42 (2015), 181-200. 1, 3, 3.7

[9] P. Cerone, S. S. Dragomir, Some new Ostrowski-type bounds for the Čebyšev functional and applications, J. Math. Inequal., 8 (2014), 159-170. 3.1, 3.2

[10] I. Csiszár, Information-type measures of difference of probability distributions and indirect observations, Studia Sci. Math. Hungar., 2 (1967), 299-318. 4.1 
[11] I. Csiszár, Information measures: a critical survey, Transactions of the Seventh Prague Conference on Information Theory, Statistical Decision Functions and the Eighth European Meeting of Statisticians, B (1978), 73-86. 4.1

[12] A. L. Gibbs, On choosing and bounding probability metrics, Int. Stat. Rev., 70 (2002), 419-435. 4

[13] L. Horváth, Đ. Pečarić, J. Pečarić, Estimations of f-and Rényi divergences by using a cyclic refinement of the Jensen's inequality, Bull. Malays. Math. Sci. Soc. (2), 42 (2019), 933-946. 4.2, 4.4

[14] K. A. Khan, T. Niaz, Đ. Pečarić, J. Pečarić, Refinement of Jensen's inequality and estimation of f-and Rényi divergence via Montgomery identity, J. Inequal. Appl., 2018 (2018), 14 pages. 4

[15] N. Levinson, Generalization of an inequality of Ky Fan, J. Math. Anal. Appl., 8 (1964), 133-134. 1

[16] F. Liese, I. Vajda, Convex Statistical Distances, BSB B. G. Teubner Verlagsgesellschaft, Leipzi, (1987). 4

[17] A. M. Mercer, A variant of Jensen's inequality, JIPAM. J. Inequal. Pure Appl. Math., 4 (2003), 2 pages. 1

[18] D. S. Mitrinović, J. E. Pečarić, A. M. Fink, Classical and New Inequalities in Analysis, Kluwer Academic Publishers Group, Dordrecht, (1993). 1, 1, 1, 2.1

[19] J. Pečarić, On an inequality on N. Levinson, Publ. Elektroteh. Fak., Univ. Beogr., Ser. Mat. Fiz., 1980 (1980), 71-74. 1, 2.1

[20] J. Pečarić, M. Praljak, A. Witkowski, Generalized Levinson's inequality and exponential conveity, Opuscula Math., 35 (2015) 397-410. 1

[21] J. Pečarić, M. Praljak, A. Witkowski, Linear operators inequality for n-convex functions at a point, Math. Inequal. Appl., 18 (2015) 1201-1217. 1

[22] J. E. Pečarić, F. Proschan, Y. L. Tong, Convex functions, partial orderings, and statistical applications, Academic Press, Boston, (1992). 1, 1, 1

[23] T. Popoviciu, Sur une inegalite de N. Levinson, Mathematica (Cluj), 6 (1969), 301-306. 1

[24] I. Sason, S. Verdú, f-divergence inequalities, IEEE Trans. Inform. Theory, 62 (2016), 5973-6006. 4

[25] I. Vajda, Theory of Statistical Inference and Information, Kluwer, Dordrecht, (1989). 4 